\title{
Spatial association between regionalizations using the information-theoretical $V$-measure
}

\author{
J. Nowosad ${ }^{a}$ and T.F. Stepinski ${ }^{a *}$ \\ ${ }^{a}$ Space Informatics Lab, University of Cincinnati, OH, USA
}

\begin{abstract}
There is a keen interest in inferring spatial associations between different variables spanning the same study area. We present a method for quantitative assessment of such associations in the case where spatial variables are either in the form of regionalizations or in the form of thematic maps. The proposed index of spatial association - called the $V$-measure - is adapted from a measure originally developed in computer science, where it was used to compare clusterings, to spatial science for comparing regionalizations. The $V$-measure is rooted in the information theory and, at its core, it is equivalent to mutual information between the two regionalizations. Here we re-introduce the $V$-measure in terms of spatial variance analysis instead of information theory. We identify three different contexts for application of the $V$-measure, comparative, associative, and derivative, and present an example of an application for each of them. In the derivative context, the $V$-measure is used to select an optimal number of regions for clustering-derived regionalizations. In effect, this also constitutes a novel way to determine the number of clusters for nonspatial clustering tasks as well. The advantage of $V$-measure over the Mapcurves method is discussed. We also use the insight from deriving the $V$-measure in terms of spatial variance analysis to point out a shortcoming of the Geographical Detector - a method to quantify associations between numerical and categorical spatial variables. The open-source software for calculating the $V$-measure accompanies this paper.
\end{abstract}

\section{KEYWORDS}

spatial association; regionalization; mutual information; clustering; Geographic Detector

\section{Introduction}

A common task in spatial data analysis is to calculate a degree to which two variables are spatially associated. Both, global measure (a single value assessment of an overall association), and local measures (association at each observation unit) are the soughtafter indicators. An approach to this task depends on the form of the data. If both variables are numerical, multivariate spatial correlation methods (Wartenberg 1985, Getis and Ord 1992, Lee 2001) are applied. If one variable is numerical and another categorical, the so-called geographical detector (Wang et al. 2010) is an appropriate 
methodology. If both variables are categorical the task is frequently referred to as a map comparison (Foody 2007).

There are two different contexts which call for map comparison. In most cases, the context is the comparison of thematic maps (for example, land cover maps of the same area at different times) where map units (often raster cells) are assigned a unique category from a relatively short list of possible themes. In thematic maps, many disjointed map units are assigned the same category. Another context is a comparison of regionalizations. A regionalization is a segmentation of the entire spatial domain (an area of interest) into a set of geographically meaningful single-connected units each having its unique name. Examples of regionalizations include maps of climate classification (Kottek et al. 2006, Peel et al. 2007, Cannon 2012, Zscheischler et al. 2012, Zhang and Yan 2014, Netzel and Stepinski 2016), maps of ecoregions (Omernik and Griffith 2014, Bailey 2014, Olson et al. 2001), and administrative maps. Note that in practice, the single-connectedness of all regions is a goal which is rarely achieved. All examples given above have some regions consisting of disjointed parts (for example, in the regionalization of the U.S. into the states, the state of Michigan consists of two disjointed parts). Thus, for the purpose of this paper, there is no difference between regionalization and the thematic map if, in the later, we consider the sets of units assigned to the same category (sometimes referred to as strata, see, for example in Wang et al. (2010) or Metzger et al. (2012)) as regions. In the rest of this paper, we will use a term regionalization to cover both contexts.

The bulk of the previous work on map comparison (Power et al. 2001, Hagen 2003, Foody 2004, Visser and DeNijs 2006) was done in the context of raster thematic maps. Such methods overlay two raster maps and perform a cell-by-cell comparison to assess the similarity between the two maps. Hargrove et al. (2006) discussed many disadvantages of such approach and proposed a map comparison based on a degree of overlap between regions in the two maps (the so-called "Mapcurves" method). Here we propose a method of assessing a degree of spatial association between regionalizations also based on regions, but, whereas Hargrove et al. (2006) rely on analyzing overlaps between regions, we rely on analyzing a variance of regions stemming from one regionalization in regions stemming from another regionalization. Our approach leverages a conceptual similarity between the problem of map comparison in geography and the problem of clustering comparison in computer science (Rosenberg and Hirschberg 2007). It also reveals a conceptual similarity between the problem of map comparison and the method of Geographical Detector (Wang et al. 2010) and points to how the Geographic Detector method could be improved.

We identify three different contexts in which a quantitative measure of association between regionalization could be used: (1) comparative, (2) associative, and (3) derivative. The comparative context involves comparing two regionalizations created to depict the same realm. One example of such context is the global mapping of climate types. Widely used map of climate types (see for example Kottek et al. (2006)) is based on the Köppen-Geiger classification scheme (Köppen 1936). Modern approaches to global climate classification (Metzger et al. 2012, Zscheischler et al. 2012, Zhang and Yan 2014, Netzel and Stepinski 2016) are based on clustering of local climates from gridded climate data (see, for example, Hijmans et al. (2005)). Using our method it is possible to assess correspondence (or lack of it) between these different approaches to climate classification. Another example of comparative context is ecoregion mapping. For the United States, we have three widely referenced delineations of ecoregions, one developed by the U.S. Environmental Protection Agency (Omernik and Griffith 2014), another developed by the U.S. Forest Service (Bailey 2014), and the third - the 
Terrestrial Ecoregions of the World - developed by Olson et al. (2001). They all are designed to depict the same realm but use different methodologies; our method can quantitatively assess a degree of similarity between those different delineations.

An associative context involves finding magnitudes of associations between a target regionalization (response variable), and a number of regionalizations corresponding to possible predictors of this target. An example of such context is a regionalization of a domain into ecoregions as a target and categorical maps of land cover, landforms, soils, and climate covering the same domain as possible predictors for ecoregions (Nowosad and Stepinski 2018b).

Finally, the derivative context relates to how some regionalizations are created. Increasingly, regionalizations are not created manually (an analyst decides the locations of the boundaries between the regions) but rather automatically via algorithmic clustering of the domain. Examples of regionalizations created via clustering include maps of global climate types (Metzger et al. 2012, Zscheischler et al. 2012, Zhang and Yan 2014, Netzel and Stepinski 2016), map of land pattern types in the U.S. (Niesterowicz and Stepinski 2013) and maps of forest types in Canada (Partington and Cardille 2013, Niesterowicz and Stepinski 2017). When creating a regionalization via clustering it is not immediately clear into how many clusters (regions) divide the domain. The computer science community has developed several heuristics to determine an "optimal" number of clusters (Rousseeuw 1987, Salvador and Chan 2004, Davies and Bouldin 1979), they all aim at minimizing dissimilarities between data instances within clusters and maximizing dissimilarities between the clusters. Our method selects the number of clusters in a spatial dataset from a different, cartographic, perspective by determining the number of regions above which the further change to regionalization - a spatial manifestation of clustering - does not change the map in a meaningful way.

\section{Methodology}

We propose to assess a degree of spatial association between regionalizations using an information-theoretical measure called the $V$-measure (Rosenberg and Hirschberg 2007). The $V$-measure originated in the field of computer science and was developed as a tool for assessing the performance of clustering algorithms. Clustering is the task of grouping a set of objects into clusters in such a way that objects in the same cluster are more similar to each other than to those in other clusters. If objects happen to have independent labels, the quality of clustering can be assessed objectively by quantifying a degree of homogeneity of labels in clusters. The $V$-measure is a particularly effective tool for such evaluation. We propose to applied the $V$-measure concept to assess a degree of association between two different regionalizations of the same domain. In the spatial context, one regionalization corresponds to clustering (a division of the domain into regions) and another corresponds to "independent labels" (a division of the domain into another set of regions).

Let's denote the area of the domain as $A$. Consider two different regionalizations of the domain. To make a further discussion more lucid we will refer to the first one as a regionalization and to the second one as a partition. The regionalization $R$ divides the domain into $n$ regions $r_{i} \mid i=1, \ldots, n$. The partition $Z$ divides the domain into $m$ zones $z_{j} \mid j=1, \ldots, m$. We use the term zone to denote a region in the second regionalization. Superposition of regionalization and partition divides the domain into up to $n \times m$ segments having areas $a_{i, j} \mid i=1, \ldots, n ; j=1, \ldots, m$ where $a_{i, j}$ is the area of the segment of the domain which belong simultaneously to the region $i$ and 
to the zone $j$. The entire area of a region $r_{i}$ is $A_{i}=\sum_{j, 1}^{m} a_{i, j}$, the entire area of a zone $z_{j}$ is $A_{j}=\sum_{i, 1}^{n} a_{i, j}$, and the area of the entire domain is $A=\sum_{j=1}^{m} \sum_{i, 1}^{n} a_{i, j}$. There are two different metrics needed for evaluation of spatial association between two regionalization, homogeneity and completeness.

Consider the following expression:

$$
\left\{\begin{array}{l}
\text { inhomogeneity of parti- } \\
\text { tion with respect to re- } \\
\text { gionalization }
\end{array}\right\}=\sum_{j=1}^{m}\left(\frac{A_{j}}{A}\right) \frac{\text { variance of regions in zone }}{j}
$$

A nominator in the fraction on the right side of eq.(1) measures an inhomogeneity of a given zone in terms of regions. This is measured in terms of the Shannon entropy (Shannon 1948):

$$
S_{j}^{\mathrm{R}}=-\sum_{i=1}^{n} \frac{a_{i, j}}{A_{j}} \log \frac{a_{i, j}}{A_{j}}
$$

If $S_{j}^{\mathrm{R}}=0$ the zone $j$ is homogeneous in terms of regions (it is a part of a single region). When the value of $S_{j}^{\mathrm{R}}$ increases the zone $j$ is increasingly inhomogeneous in terms of regions (it overlays an increasing number of regions). Eq.(2) quantifies the level of this inhomogeneity or a variance of regions in zone $j$. However, we are not so much interested in the absolute value of the zone inhomogeneity as in its value relative to the inhomogeneity of the entire domain with respect to regions (a denominator in the fraction on the right side of eq.(1)). This is because for the partition to be associated with regionalization the regions should be co-located with the zones, so regions within zones should have less variance than within the entire domain. The dispersion of regions in the entire domain is also given by the Shannon entropy:

$$
S^{\mathrm{R}}=-\sum_{i=1}^{n} \frac{A_{i}}{A} \log \frac{A_{i}}{A}
$$

An overall inhomogeneity of partition with respect to regionalization is $\sum_{j=1}^{m}\left(A_{j} / A\right)\left(S_{j}^{\mathrm{R}} / S^{\mathrm{R}}\right)$, an area-weighted average of $S_{j}^{\mathrm{R}} / S^{\mathrm{R}}$ ratios calculated over all zones (see eq.(1)). The value of an overall inhomogeneity changes from 0 in the perfectly homogeneous case (each zone is within a single region) to 1 when each zone has the same composition of regions as the entire domain. The homogeneity metric suppose to be an increasing function of an average homogeneity of zones with respect to regions, therefore it is defined as

$$
h=1-\sum_{j=1}^{m}\left(A_{j} / A\right)\left(S_{j}^{\mathrm{R}} / S^{\mathrm{R}}\right)
$$

and it has a range between 0 and 1 .

Note that homogeneity metric is not sufficient to assess a degree of association between regionalization and partitioning. The high value of $h$ assures that zones are 
homogeneous with respect to regions, but it does not assure that regions are homogeneous with respect to zones. For example, when a single region extends over multiple zones, each zone will be homogeneous but there will be no association between the regionalization and partitioning. Therefore, we need to calculate a homogeneity of regions with respect to zones. This metric - called completeness and denoted by $c$ - is calculated analogously to homogeneity but with the roles of regions and zones reversed.

$$
\begin{aligned}
\left.\begin{array}{l}
\text { inhomogeneity of region- } \\
\text { alization with respect to } \\
\text { partition }
\end{array}\right\} & =\sum_{i=1}^{n}\left(\frac{A_{i}}{A}\right) \frac{\text { variance of zones in region }}{i} \\
S_{i}^{\mathrm{Z}} & =-\sum_{j=1}^{m} \frac{a_{i, j}}{A_{i}} \log \frac{a_{i, j}}{A_{i}} \\
S^{\mathrm{Z}} & =-\sum_{j=1}^{m} \frac{A_{j}}{A} \log \frac{A_{j}}{A} \\
c & =1-\sum_{i=1}^{n}\left(A_{i} / A\right)\left(S_{i}^{\mathrm{Z}} / S^{\mathrm{Z}}\right)
\end{aligned}
$$

Completeness, like the homogeneity, has the range between 0 and 1 and is an increasing function of average homogeneity of regions with respect to zones. The single, overall measure of spatial association between regionalization and partition is called the $V$ measure (Rosenberg and Hirschberg 2007) and is given by the (optionally weighted) harmonic mean of homogeneity and completeness:

$$
V_{\beta}=\frac{(1+\beta) h c}{(\beta h)+c}
$$

If $\beta>1$ than completeness is weighted more strongly than homogeneity but if $\beta<1$ than homogeneity is weighted more strongly. $V_{\beta}$ has a range between 0 (no spatial association) and 1 (a perfect association). Note that if we change the roles of regionalization and partitioning so the regionalization provides the zones and partitioning provides the regions we don't need to recalculate the measures $h$ and $c$ as the $h_{\text {new }}=c$ and $c_{\text {new }}=h$. The $V$-measure will remain the same if $\beta=1$ (which is a default value).

Fig. 1 illustrates the procedure of calculating $h, c$, and $V_{1}$ using a simple example. These three quantities are the global measures of association between two regionalizations. $V_{1}$ is an overall global measure to be used when a single number assessment of association is required. As a pair, the values of $h$ and $c$ provide more information than $V_{1}$ alone. Ratios $S_{j}^{\mathrm{R}} / S^{\mathrm{R}}, j=1, \ldots, m$ and $S_{i}^{\mathrm{Z}} / S^{\mathrm{Z}}, i=1, \ldots, n$ are the local measures of association between the two regionalization. They could be used to map a degree of local correspondence between two regionalizations. 


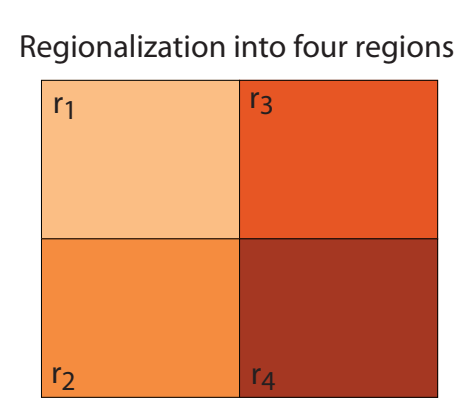

Partitioning into three zones

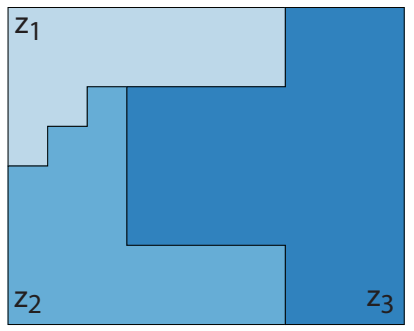

Inhomogeneity of regions in zones

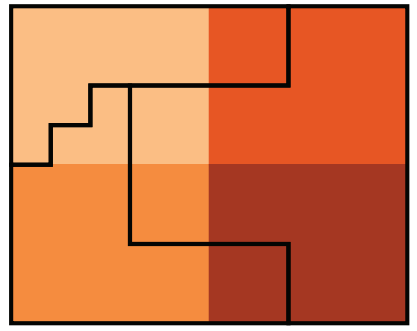

Inhomogeneity of zones in regions

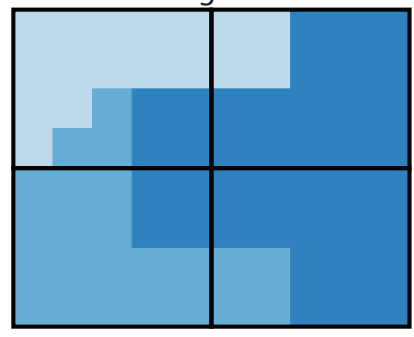

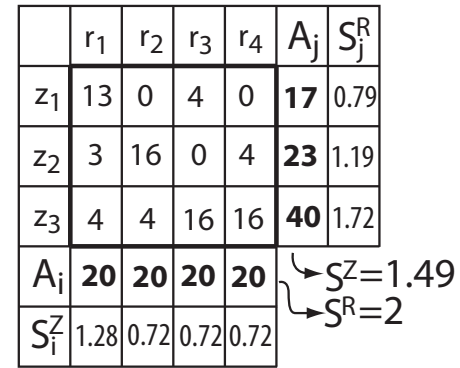

$h=1-\sum_{j=1}^{3} \frac{A_{j}}{A} \frac{S_{j}^{R}}{S^{R}}=0.32$ $c=1-\sum_{i=1}^{4} \frac{A_{i}}{A} \frac{S_{i}^{Z}}{S^{Z}}=0.42$ $\mathrm{V}_{1}=\frac{\mathrm{hc}}{\mathrm{h}+\mathrm{c}}=0.36$

Figure 1. An example illustrating an assessment of the association between two regionalizations. The red regionalization segments a rectangular domain into four regions, The blue regionalization (partition) segments the same domain into three regions (zones). The variance of red regions in the three zones and the variance of blue zones in four regions are shown. Values of $a_{i, j}$ (in arbitrary units) are given in the part of the table enclosed by the thick-edged rectangle.

\subsection{Software}

We wrote an open-source $\mathrm{R}$ package implementing the $V$-measure (Nowosad and Stepinski 2018a). The package, called SABRE (Spatial Association Between REgionalizations), is designed to work with vector (shapefile) input data. Given two vector maps, SABRE calculates values of $V_{\beta}, h$, and $c$ to be used as the global assessment of association between the two maps. It also returns maps of local associations utilizing the values $S_{j}^{\mathrm{R}} / S^{\mathrm{R}}, j=1, \ldots, m$, and $S_{i}^{\mathrm{Z}} / S^{\mathrm{Z}}, i=1, \ldots, n$. SABRE also implements the Mapcurves method (Hargrove et al. 2006) for vector maps. 


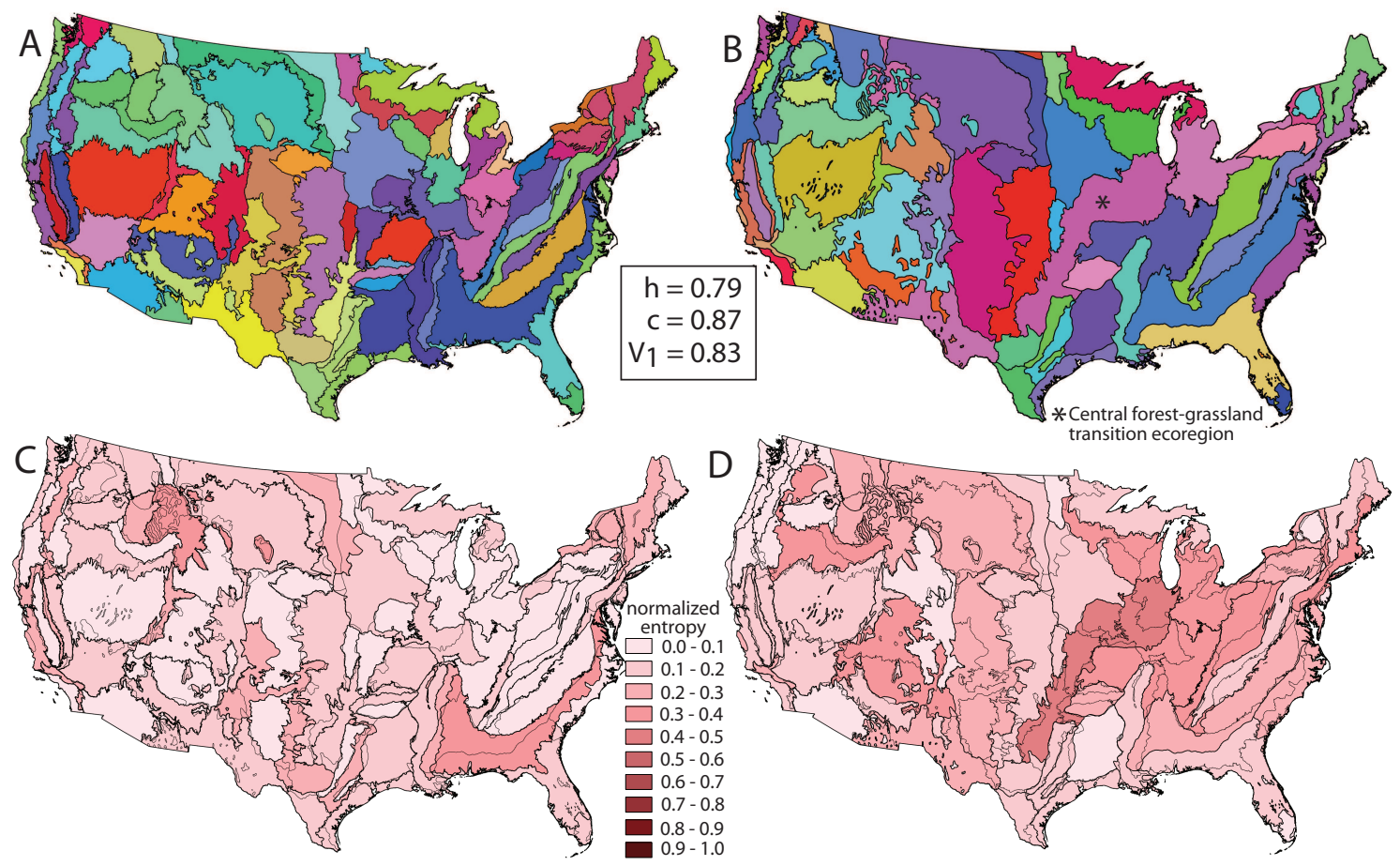

Figure 2. Spatial association between two ecoregionalizations of the conterminous U.S. The top row shows the EPA Level III map of ecoregions (A) and the TEW map of ecoregions (B). In both maps different ecoregions are shown by random colors. The bottom row shows a map of inhomogeneity of EPA ecoregions in terms of TEW ecoregions (C) and a map of inhomogeneity of TEW ecoregions in terms of EPA ecoregions. Inhomogeneity (variance) is measured by normalized Shannon entropy.

\section{Applications}

In this section we present examples of how the $V$-measure may be used in each of the three contexts identified in the Introduction: to compare two regionalization, to calculate a degree of associative between response map and maps of factor variables, and to decide on the number of regions in regionalization obtained by means of a clustering algorithm.

\subsection{Comparing ecoregionalizations of the United States}

Ecoregions are the result of a division of land into areal units of a homogeneous ecosystem which contrast from surroundings. The U.S. Environmental Protection Agency (EPA) delineated ecoregions in the conterminous U.S. at four hierarchical levels of precision (Omernik 1987, Omernik and Griffith 2014). For the purpose of this demonstration, we use EPA Level III map as the first regionalization; it delineates the U.S. into $n=85$ regions (see Fig. 2A). For comparison we use the Terrestrial Ecoregions of the World (TEW) map (Olson et al. 2001) restricted to boundaries of the conterminous U.S. as the second regionalization; it delineates the U.S. into $m=72$ zones (see Fig. 2B). Both maps suppose to reflect the same realm but were constructed us- 
ing different methodologies. The EPA map was constructed by analyzing the patterns and composition of biotic and abiotic phenomena that affect or reflect differences in ecosystems. The TEW map is based on the synthesis of previous biogeographical studies (possibly including the EPA maps). Visual comparison of Figs. 2A and 2B reveals the overall similarity between the two maps, but also local differences between them. The $V$-measure method can quantify the similarity and depict the locations of greatest differences between the two maps.

Using SABRE we calculated $h=0.79, c=0.87$, and $V_{1}=0.83$ as global measures of association between EPA and TEW maps. Recall from section 2 that $h$ measures an average homogeneity of TEW zones with respect to EPA regions (eq. 4 and Fig. 2D) and $c$ measures a homogeneity of EPA regions with respect to TEW zones (eq. 8 and Fig. 2C). Visually, the map in Fig. 2C appears to be more homogeneous than the map in Fig. 2D in agreement with quantitative assessment $c>h$. This is because, there are more EPA ecoregions than TEW ecoregions, so it is more likely that TEW ecoregions cross through multiple EPA ecoregions than the vice versa. However, overall, the two maps are highly associated as indicated by the high value of $V_{1}$. The two inhomogeneity maps (Figs. 2C and 2D) identify locations where the two maps differ. The biggest difference between the two maps is in the middle of the country where a single TEW ecoregion (named "Central forest-grassland transition") intersect 12 different EPA ecoregions.

\subsection{Associations between a map of ecoregions and its factors}

As we mentioned in the previous subsection EPA regionalization of the conterminous U.S. is based on the analysis of patterns and composition of biotic and abiotic factors including geology, landforms, soils, vegetation, climate, land cover, wildlife, and hydrology. Here we demonstrate the utility of the $V$-measure to assess a degree of correspondence between the EPA Level III map of ecoregions and maps of four such factors: land cover, soils, landforms, and climate. For clarity, we restrict this demonstration to a territory of a single state - New Mexico.

The factors are all in the form of thematic (categorical) maps. We use the European Space Agency (ESA) Climate Change Initiative (CCI) $300 \mathrm{~m}$ resolution global land cover map (CCI-LC 2015) which classifies land cover worldwide into 22 classes. Soil data is provided by the $250 \mathrm{~m}$ resolution global SoilGrids (Hengl et al. 2017) reclassified to 12 orders. Landforms data is a $250 \mathrm{~m}$ resolution classification of landforms into 17 classes (Karagulle et al. 2017). Finally, the climate data is provided by clustering a set of bioclimatic variables at worldwide climatic grid into 37 classes (Metzger et al. 2012).

Table 1. Spatial associations between the EPA map of ecoregions in the state of New Mexico and its biotic and abiotic factors

\begin{tabular}{l|llll||llll}
\hline & \multicolumn{4}{|c||}{ Thematic maps } & \multicolumn{4}{c}{ Segmentations } \\
\hline Factor & $m$ & $h$ & $c$ & $V_{1}$ & $m$ & $h$ & $c$ & $V_{1}$ \\
\hline Land cover & 17 & 0.25 & 0.37 & 0.30 & 188 & 0.72 & 0.35 & 0.47 \\
Soils & 11 & 0.20 & 0.31 & 0.24 & 219 & 0.75 & 0.34 & 0.47 \\
Landforms & 15 & 0.20 & 0.18 & 0.19 & 775 & 0.87 & 0.27 & 0.41 \\
Bioclimates & 11 & 0.24 & 0.43 & 0.31 & $\mathrm{~N} / \mathrm{A}$ & $\mathrm{N} / \mathrm{A}$ & $\mathrm{N} / \mathrm{A}$ & $\mathrm{N} / \mathrm{A}$ \\
\hline$m$ - number & of zones or segments, $h$ - homogeneity, $c$ - completeness, \\
$V_{1}-V$-measure
\end{tabular}

Fig. 3 shows a map of EPA level III ecoregions and the maps of the four factors within the state of New Mexico. We use SABRE to calculate values of $h, c$, and $V_{1}$ to assess a spatial association between EPA ecoregionalization (eight ecoregions within 

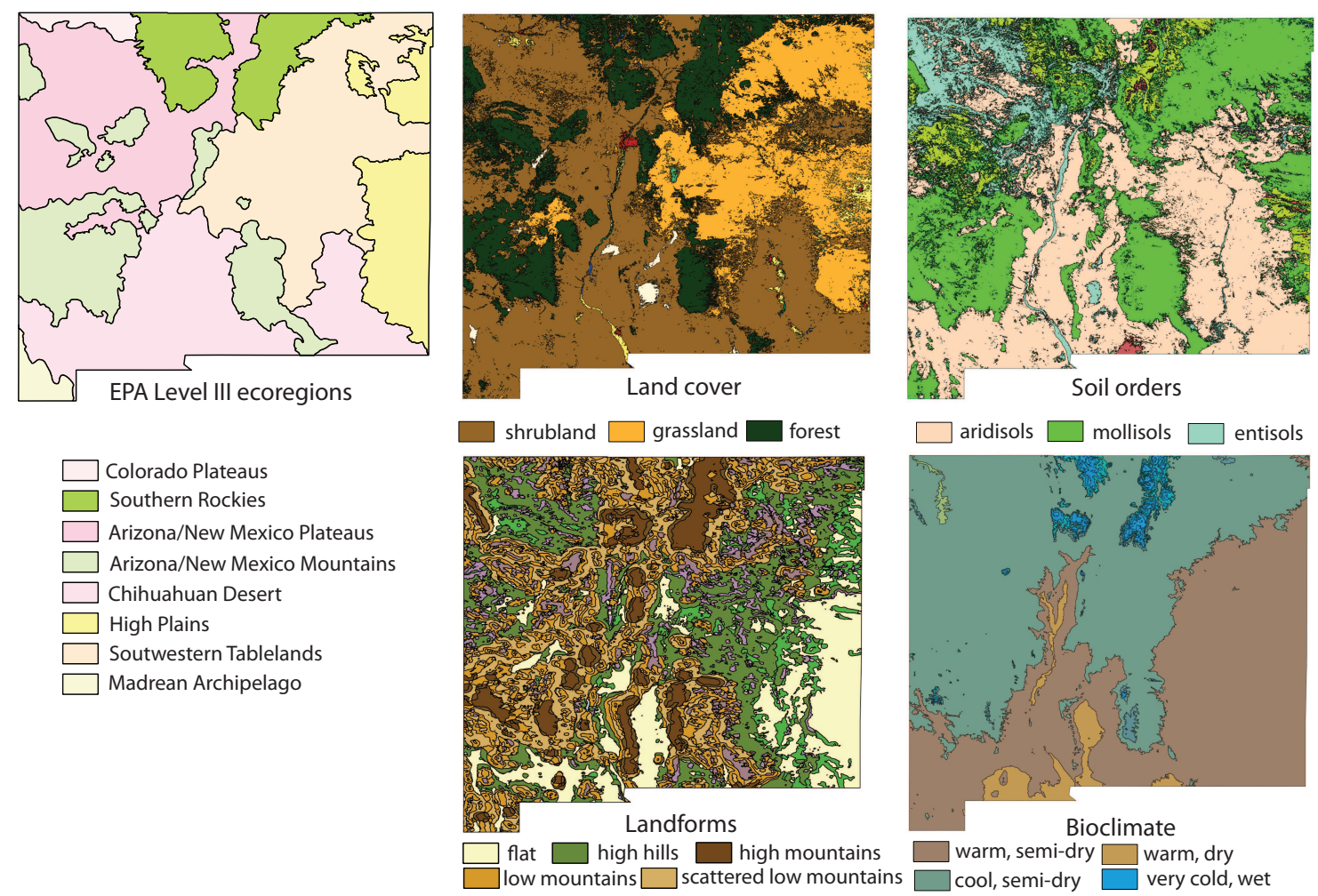

Figure 3. EPA Level III ecoregions in the state of New Mexico and the maps of four factors influencing a delineation of these ecoregions. Legends for the maps of the factors show only dominant categories.

the state of New Mexico) and a thematic map of each factor. The "Thematic maps" section of Table 1 shows the results. The first column (denoted by $m$ ) in this section lists the number of categories in a given map present within the state of New Mexico; this is also a number of zones in the factor map. The values of $h$ measure average homogeneity of factors' zones with respect to ecoregions and the values of $c$ measure homogeneity of ecoregions with respect to factors' zones. Note that values of $c$ tends to be higher than the values of $h$ (except for landforms) indicating that ecoregions are more homogeneous with respect land cover, soils, and, in particular, the climate, than categories of factors are homogeneous with respect to ecoregions (for example, multiple ecoregions are found within a climate category "cool, semi-dry"). Overall, associations between the map of ecoregions and thematic maps of individual factors are low as indicated by small values of $V_{1}$.

However, it is important to note that EPA ecoregions were not constructed on the basis of homogeneity of factors categories, but rather on the basis of homogeneity of patterns of factor categories. We used a method for pattern-based segmentation of thematic maps (Jasiewicz et al. 2017, Nowosad and Stepinski 2018b) to calculate segmentations of the area of New Mexico with respect to homogeneity of patterns of land cover categories, soil classes, and landforms categories. The climate zones have too large spatial extent for calculation of pattern at the scale of the state of New Mexico. Fig. 4 (top row) shows the segmentations. Note that there are much more segments 
than ecoregions. This is because segments are the results of machine delineation which painstakingly keep track of all changes in a pattern, whereas ecoregions are the result of manual mapping which is much more generalized. The middle row of Fig. 4 shows inhomogeneity maps of ecoregions with respect to segments and the bottom row of Fig. 4 shows inhomogeneity maps of segments with respect to ecoregions.
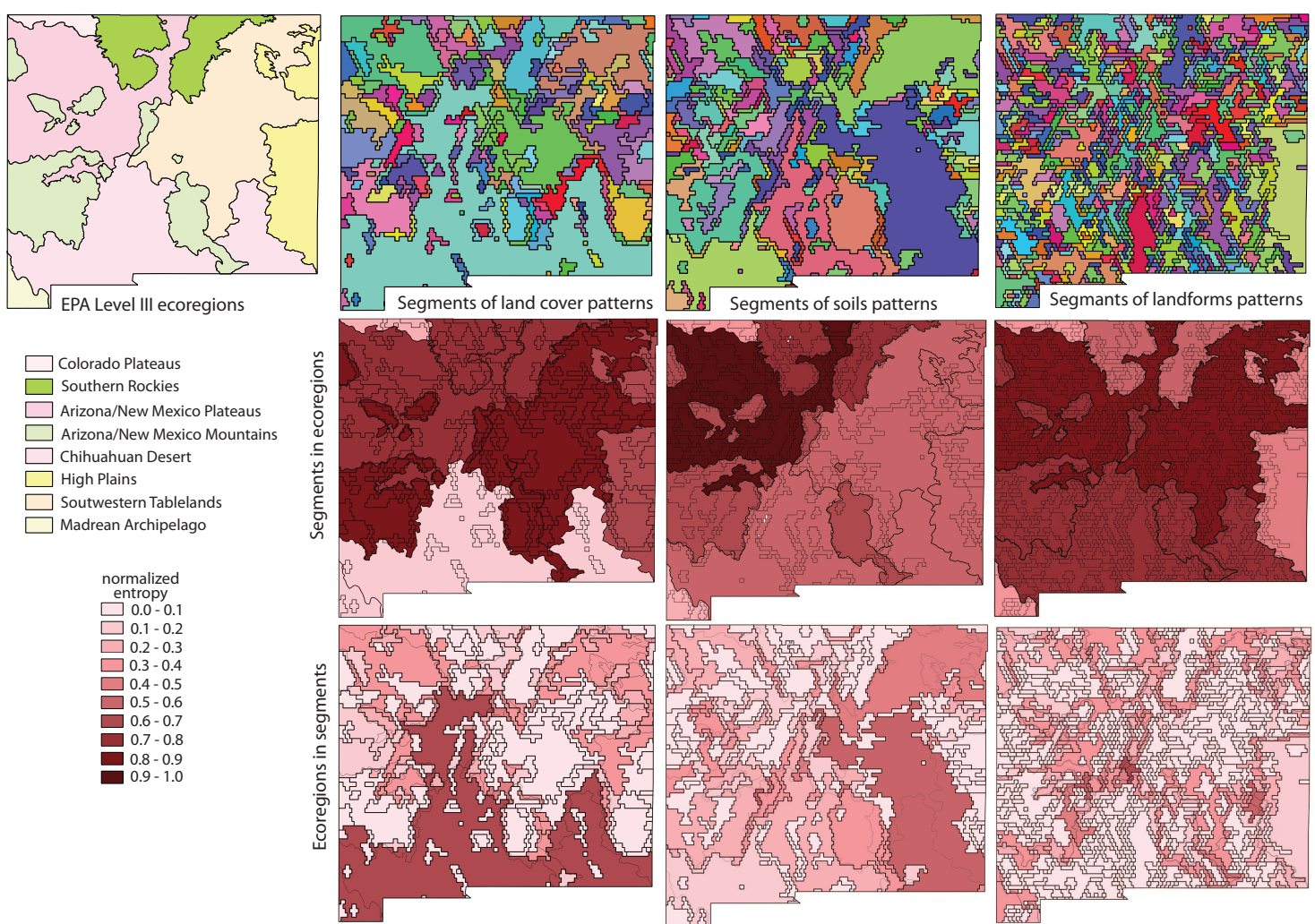

Figure 4. (Top row) Segmentations of influencing factors for delineation of ecoregions with respect to homogeneity of patterns of their categories. Segments are indicated by random colors. (Middle row) Inhomogeneity maps of ecoregions with respect to segments. (Bottom row) Inhomogeneity maps of segments with respect to ecoregions.

We calculated values of $h, c$, and $V_{1}$ to assess a spatial association between EPA ecoregionalization and the three segmentations. The "Segmentations" section of Table 1 shows the results with $m$ indicating the number of segments. Note that the values of $h$ are high because small segments usually are contained within a single ecoregion, but the values of $c$ are lower because larger ecoregions usually contain several segments. Overall, associations between the map of ecoregions and maps delineating homogeneous patterns of factors are relatively high (as indicated by values of $V_{1}$ ), and, in any case, significantly higher than associations between the map of ecoregions and thematic maps of individual factors. 


\subsection{Selecting a number of clusters in regionalizations stemming from clustering}

A number of studies had proposed algorithmic regionalization by means of clustering a large number of small local areal units (elements) into a small number of larger regions (clusters of elements) based on similarity of features. This includes clustering local climates (Metzger et al. 2012, Zhang and Yan 2014, Netzel and Stepinski 2016) to obtain climatic zones, clustering local environmental conditions to obtain ecoregions (Hargrove and Hoffman 2005), and clustering local landscapes to obtain regions of the uniform pattern of land cover (Partington and Cardille 2013, Niesterowicz and Stepinski 2013, Niesterowicz et al. 2016). All these studies encounter the problem of selecting a number of clusters and thus the number of regions in the resultant map. The number of regions is estimated using methods developed for non-spatial clustering (Rousseeuw 1987, Salvador and Chan 2004, Davies and Bouldin 1979). The $V$-measure offers a different, distinctly spatial method for estimating the number of regions resulting from clustering.

In the proposed method a sequence of clusterings with a consecutively increasing number of clusters is calculated. Next, for each clustering, a value of $V$-measure between it and the subsequent clustering is calculated. This value indicates a degree of similarity between maps stemming from the two clusterings. For clusterings with a small number of clusters the maps are different and $V_{1}$ (map1, map2) is relatively small. As the number of clusters increases, the two consecutive maps are becoming more similar and $V_{1}$ (map1, map2) increases. The map with an optimal number of regions (clusters) is the one for which the $V_{1}$ achieves the maximum value.
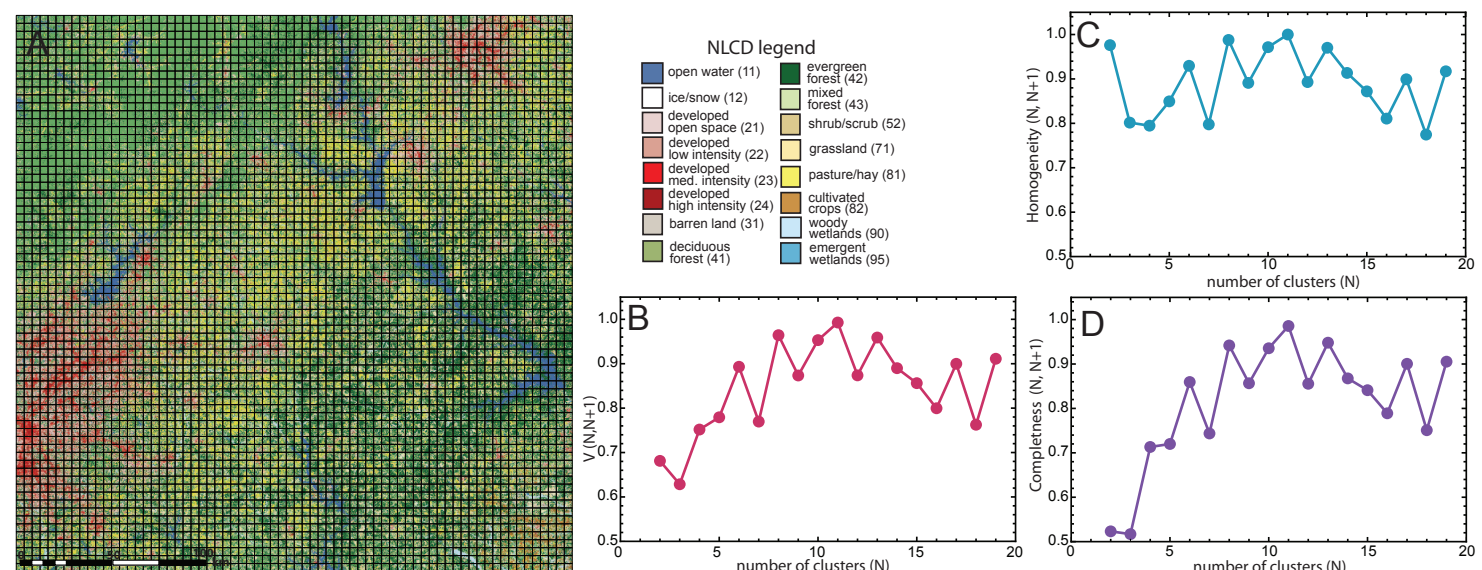

Figure 5. NLCD 2011 over the study area located around Atlanta, Georgia tessellated into 4,900 local landscapes, each having size of $3 \mathrm{~km} \times 3 \mathrm{~km}$. Different colors indicate different land cover categories as described by the legend. (Right) Results of $V$-measure analysis using consecutive regionalizations with increasing number of regions, (B) $V_{1},(\mathrm{C})$ homogeneity, (D) completeness. 

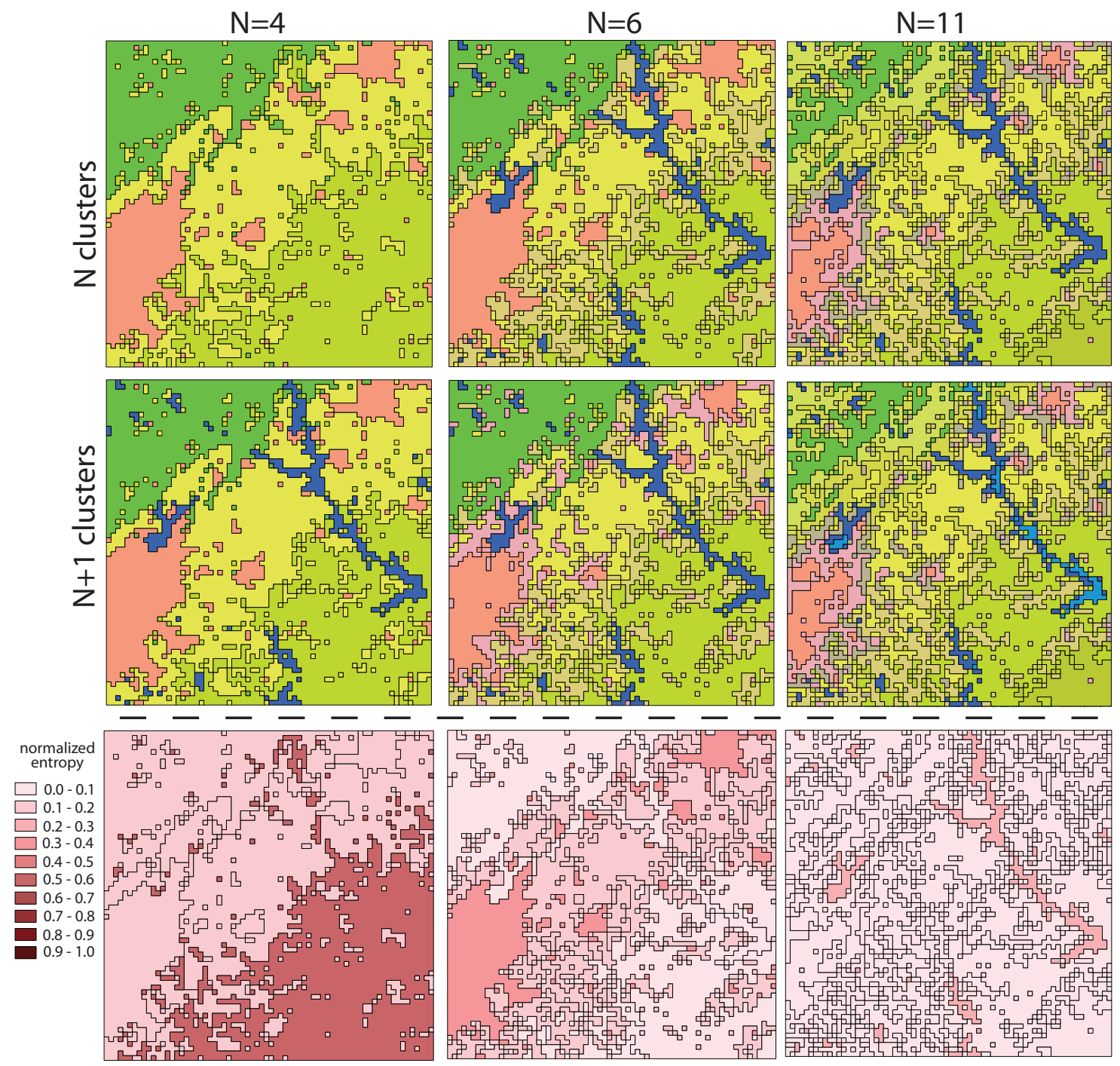

Figure 6. Examples of regionalizations of the Atlanta study area. Each column consists of a regionalization with a given number of regions (top) and a regionalization with one additional region (middle). The inhomogeneity map of regions in the top map with respect to regions in the middle map is given at the bottom of the column. Colors in the top and middle rows indicate different regions.

To demonstrate the proposed method we consider a problem of regionalization of land cover patterns. We start with $210 \mathrm{~km} \times 210 \mathrm{~km}$ study area located around Atlanta, Georgia, with land cover represented by the $30 \mathrm{~m}$ resolution National Land Cover Dataset 2011 (NLCD 2011). We tessellate this area into 4,900 square-sized local landscapes (each consisting of $100 \times 100$ NLCD cells) as shown in Fig. 5A. Next, we cluster local landscapes using a method described by Niesterowicz et al. (2016) but using a non-hierarchical Partitioning Around Medoids (PAM) clustering algorithm (Kaufman and Rousseeuw 1987). We performed 19 clustering assuming number of clusters from $N=2$ to $N=20$. Fig. $5 \mathrm{~B}$ shows dependence of $V_{1}$ (map1,map2), were map1 is a regionalization with $N$ regions and map2 is a regionalization with $N+1$ regions. The 
value $V_{1}$ achieves maximum at $N=11$, thus we selected a map with 11 regions as the optimal regionalization.

The top row of Fig. 6 shows three out of 19 regionalizations of the Atlanta study area, using $N=4, N=6$ and $N=11$ regions, respectively. Middle row of Fig. 6 shows corresponding subsequent regionalizations $(N=5, N=7$ and $N=12)$. The bottom row of Fig. 6 shows inhomogeneity maps of $N$ regions in the top map in terms of $N+1$ regions in the middle map. Changing the number of regions from $N=4$ to $N=5$ results in a separation of the blue region from the light-green region, thus the light-green region is relatively inhomogeneous with respect to other three regions in the $N=4$ regionalization (see the rightmost column in Fig. 6). Because the light-green region occupies a large portion of the study area, its inhomogeneity enters the calculation of the $V$-measure with the high weight resulting in a relatively low value of $V_{1}$. Changing the number of regions from $N=11$ to $N=12$ results in dividing the blue region into two different regions (see the leftmost column in Fig. 6). However, because the blue region in $N=11$ regionalization occupies a small portion of the study area, its inhomogeneity enters into the calculation of the $V$-measure with a small weigh resulting in a relatively high value of $V_{1}$.

\section{Discussion and Conclusions}

The $V$-measure method for assessing a degree of spatial association between regionalizations has two advantages over the Mapcurves (Hargrove et al. 2006) method. First, the $V$-measure has a clear interpretation in terms of the information-theoretical notion of mutual information. If we consider the two regionalization maps as random variables than the mutual information $I$ (map1, map2) measures the amount of information contained in map1 about map2 (and vice versa) and thus it assesses the degree of dependence between the two maps. Note that the homogeneity $h$, given by eq. 4 , is equal to $I$ (map1, map2) $/ S^{\mathrm{R}}$, and the completeness $c$, given by eq. 8 , is equal to $I$ (map1, map2) $/ S^{\mathrm{Z}}$. We use normalized variants of mutual information because we want values of $h$ and $c$, and, ultimately, the value of $V_{\beta}$, to be in the range between 0 and 1 . However, at its core, the $V$-measure is a mutual information between the two maps. Note that mutual information is symmetric $(I(\operatorname{map} 1$, map2 $)=I(\operatorname{map} 2, \operatorname{map} 1))$ and the values of $h$ and $c$ are different only because of different normalizations.

Second, $V$-measure provides more precise information than Mapcurves. $V_{\beta}=1$ only if the two regionalizations are identical, whereas Mapcurves score equal to 1 every time one regionalization is a subdivision of the second regionalization. This is because although Mapcurves considers two goodness-of-fit scores (which are conceptually rough equivalents of ours $h$ and $c$ ) it only uses the larger one as an overall score. By using instead the harmonic mean of both scores (like we do) the precision of the Mapcurves method could be improved.

The $V$-measure also provides an insight into a shortcoming of the Geographical Detector method (Wang et al. 2010). Since its introduction in 2010, the Geographical Detector method has been popular, especially in the field of health geography. It is designed to assess a spatial association between a numerical variable and a categorical variable. Typically, the numerical variable is a response variable $(G)$ whereas the categorical variable is a potential determinant $(D)$ or "risk factor." In Geographical Detector method, the spatial association index is called the power of determinant $P(D, G)$. 


$$
P(D, G)=1-\sum_{k=1}^{K}\left(n_{k} / n\right)\left(\sigma_{k} / \sigma\right)
$$

where $K$ is the number of zones in the categorical variable $D, n_{k}$ is the number of measurements of $G$ within a zone $k, n=\sum_{k, 1}^{K} n_{k}$ is the number of all measurements of $G$ in the entire domain, $\sigma_{k}$ is a variance of variable $G$ within a zone $k$, and $\sigma$ is a variance of variable $G$ in the entire domain. Note that the mathematical form of $P(D, G)$ is identical to mathematical forms of $h$ (eq. 4) and $c$ (eq. 8). The only difference is that in $h$ and $c$ the variance is calculated using the Shannon entropy because the variable is categorical.

The problem with the Geographical Detector method is that the assessment of association is performed using only a relative homogeneity of variable $G$ in zones of $D$, but no attempt is made to assess the relative homogeneity of $D$ with respect to $G$. This is because the variable $G$ is numerical and does not naturally form zones. However, this leaves open the possibility that the assessment of the spatial association between $G$ and $D$ may be inaccurate if similar values of $G$ extend over multiple zones of $D$. In such case, the Geographical Detector method will incorrectly indicate the high spatial association. If there is a large number of $G$ measurements, a solution would be to first segment the domain with respect to homogeneity of $G$ values and then perform the assessment of the spatial association between $D$ and segmentation of $G$ using the $V$-measure.

We have identified three different contexts for application of $V$-measure and, in section 3, we gave a specific example of an application in each of these contexts. Perhaps the context of finding an optimal number of regions for clustering-based regionalization is the most interesting inasmuch as it is an original idea - a new way to determine the number of clusters which is applicable not only to the spatial domain but also to non-spatial clusterings as well.

The reason why the $V$-measure works for determining an optimal number of regions is as follows. If the number of regions is too small the regions are strongly inhomogeneous and an additional region is likely to significantly change the configuration of regionalization to improve the homogeneity of the regions. This significant change results in the small value of $V_{1}$ (left part of Fig. 5B). If the number of regions is too large the regions are almost homogeneous and an additional region forces an unnecessary change in the configuration of regionalization. This also results in a relatively small value of $V_{1}$ (left part of Fig. 5B). If the number of regions is close to being optimal an additional region causes only a small adjustment to the configuration of regionalization resulting in a high value of $V_{1}$. The same reasoning applies to a non-spatial clustering, except the results cannot be visualized in the form of the map as in Fig. 6 . Thus, we started by leveraging our familiarity with non-spatial clustering methods to propose using $V$-measure for spatial analysis, but in the process, our application of $V$-measure for regionalizations suggested a new method for determining the number of clusters in non-spatial cases as well.

\section{Acknowledgements}

This work was supported by the University of Cincinnati Space Exploration Institute. 


\section{References}

Bailey, R.G., 2014. Ecoregions: The ecosystem geography of the oceans and continents. Second Edition. Springer.

Cannon, A.J., 2012. Hydrology and Earth System Sciences. Köppen versus the computer: comparing Köppen-Geiger and multivariate regression tree climate classifications in terms of climate homogeneity, 16(1), 217-229.

Davies, D.L. and Bouldin, D.W., 1979. A cluster separation measure. IEEE transactions on pattern analysis and machine intelligence, 1(2), 224-227.

Foody, G.M., 2004. Thematic map comparison. Photogrammetric Engineering 6 Remote Sensing, 70(5), 627-633.

Foody, G.M., 2007. Map comparison in gis. Progress in Physical Geography, 31(4), 439-445.

Getis, A. and Ord, K., 1992. The analysis of spatail association by use of distance statistics. Geographical Analysis, 24, 189-206.

Hagen, A., 2003. Fuzzy set approach to assessing similarity of categorical maps. Int. J. Geographical Information Science, 17, 235-249.

Hargrove, W.W. and Hoffman, F.M., 2005. Potential of multivariate quantitative methods for delineation and visualization of ecoregions. Environmental Management, 34(1), 39-60.

Hargrove, W.W., Hoffman, F.M., and Hessburg, P.F., 2006. Mapcurves: a quantitative method for comparing categorical maps. Journal of Geographical Systems, 8(2), 187-208.

Hengl, T., et al., 2017. SoilGrids250m: Global gridded soil information based on machine learning. PloS One, 12(2), e0169748.

Hijmans, R.J., et al., 2005. Very high resolution interpolated climate surfaces for global land areas. International Journal of Climatology, 25(15), 1965-1978.

Jasiewicz, J., Stepinski, T.F., and Niesterowicz, J., 2017. Multi-scale segmentation algorithm for pattern-based partitioning of large categorical rasters. Computers $\&$ Geosciences, submitted, preprint at EarthArXiv.

Karagulle, D., et al., 2017. Modeling global Hammond landform regions from 250-m elevation data. Transactions in GIS, 21(5), 1040-1060.

Kaufman, L. and Rousseeuw, P., 1987. Statistical Data Analysis Based on the L1 Norm and Related Methods. In: Y. Dodge, ed. Clustering by means of medoids. North-Holland, 405416.

Köppen, W., 1936. Das geographische System der Klimate. In: W. Köppen and R. Geiger, eds. Handbuch der Klimatologie. Gebrder Borntraeger, Berlin, 1-44.

Kottek, M., et al., 2006. World map of the Köppen-Geiger climate classification updated. Meteorologische Zeitschrift, 15(3), 259-263.

Lee, S.I., 2001. Developing a bivariate spatial association measure: an integration of Pearson's r and Moran's I. Journal of Geographical Systems, 3(4), 369-385.

Metzger, M.J., et al., 2012. A high-resolution bioclimate map of the world: a unifying framework for global biodiversity research and monitoring. Global Ecology and Biogeography, $22(5), 630-638$.

Netzel, P. and Stepinski, T.F., 2016. On Using a Clustering Approach for Global Climate Classification. Journal of Climate, 29(9), 3387-3401.

Niesterowicz, J. and Stepinski, T.F., 2017. Pattern-based, multi-scale segmentation and regionalization of EOSD land cover. Int. Journal of Applied Earth Observation and Geoinformation, 62, 192-200.

Niesterowicz, J., Stepinski, T.F., and Jasiewicz, J., 2016. Unsupervised regionalization of the United States into landscape pattern types. International Journal of Geographical Information Science, 30(7), 1450-1468.

Niesterowicz, J. and Stepinski, T.F., 2013. Regionalization of multi-categorical landscapes using machine vision methods. Applied Geography, 45, 250-258.

Nowosad, J. and Stepinski, T.F., 2018a. sabre: Spatial Association Between REgionalizations. R package version 0.2.0, Available from: https://github.com/Nowosad/sabre.

Nowosad, J. and Stepinski, T.F., 2018b. Towards machine ecoregionalization of Earth's land- 
mass using pattern segmentation method. International Journal of Applied Earth Observation and Geoinformation, 69, 110-118.

Olson, D.M., et al., 2001. Terrestrial Ecoregions of the World: A New Map of Life on Earth A new global map of terrestrial ecoregions provides an innovative tool for conserving biodiversity. BioScience, 51(1), 933-938.

Omernik, J.M., 1987. Ecoregions of the conterminous United States. Ann. Assoc. Am. Geogr., $77(1), 118-125$.

Omernik, J.M. and Griffith, G.E., 2014. Ecoregions of the conterminous United States: evolution of a hierarchical spatial framework. Environmental management, 54(6), 1249-1266.

Partington, K. and Cardille, J.A., 2013. Uncovering dominant land-cover patterns of Quebec: Representative landscapes, spatial clusters, and fences. Land, 2(4), 756-773.

Peel, M.C., Finlayson, B.L., and McMahon, T.A., 2007. Updated world map of the KöppenGeiger climate classification. Hydrol. Earth Syst. Sci., 11, 1633-1644.

Power, C., Simms, A., and White, R., 2001. Hierarchical fuzzy pattern matching for the regional comparison of land use maps. Int. J. of Geographical Information Science, 15(1), 77-100.

Rosenberg, A. and Hirschberg, J., 2007. V-Measure: A Conditional Entropy-Based External Cluster Evaluation Measure. In: Joint Conference on Empirical Methods in Natural Language Processing and Computational Natural Language Learning. 410-420.

Rousseeuw, P.J., 1987. Silhouettes: a graphical aid to the interpretation and validation of cluster analysis. Journal of Computational and Applied Mathematics, 20, 53-65.

Salvador, S. and Chan, P., 2004. Determining the number of clusters/segments in hierarchical clustering/segmentation algorithms. In: 16th IEEE International Conference on Tools with Artificial Intelligence, 2004. 576-584.

Shannon, C.E., 1948. A mathematical theory of communication. Bell System Technical Journal, $27,379-423$

Visser, H. and DeNijs, T., 2006. The map comparison kit. Environmental Modelling \& Software, $21(3), 346-358$.

Wang, J.F., et al., 2010. Geographical Detectors-Based Health Risk Assessment and its Application in the Neural Tube Defects Study of the Heshun Region, China. International Journal of Geographical Information Science, 24(1), 107-127.

Wartenberg, D., 1985. Multivariate spatial correlation: a method for exploratory geographical analysis. Geographical Analysis, 17(4), 263-283.

Zhang, X. and Yan, X., 2014. Spatiotemporal change in geographical distribution of global climate types in the context of climate warming. Climate Dynamics, 43(3-4), 595-605.

Zscheischler, J., Mahecha, M.D., and Harmeling, S., 2012. Climate classifications: the value of unsupervised clustering. Procedia Computer Science, 9, 897-906. 